\author{
Alessandro Cupri \\ (dottorando di ricerca in Law and Pluralism nell'Università degli Studi di Milano- \\ Bicocca, Dipartimento di Giurisprudenza)
}

\title{
L'economia del Profeta: la finanza islamica e i fondamenti religiosi del diritto islamico dei contratti Shari'ah Compliant *
}

\author{
The Prophet's Economy: Islamic Finance and the Religious Foundations of Islamic Law \\ of Shari'ah Compliant Contracts
}

\begin{abstract}
The growing presence of Muslim believers in Europe has increasingly led to heated debates also at the doctrinal level. Legal and economic literature, particularly, has recently emphasised the importance that inclusive, effective and efficient financial regulation can have on the degree of integration of the Islamic communities present in non-Muslim contexts. Since the Shari'ah dictates a series of behavioural rules in different sectors of the believer's life, the Koran and the narratives of the Prophet (hadith) offer a series of prescriptions also in economic matters. With the aim of outlining the religious and juridical reasoning of the so-called halal economic dimension, this paper first examines the economic principles of Islamic finance: the prohibition of ribà (interest), the prohibition of ghàrar (uncertainty) and that of maysir (speculation). It then provides a recognition aimed at finding a general theory of Islamic contracts. Finally, it reconstructs the discipline of the mudàraba and musharàka participatory contracts. These two forms of contracts represent a product of Islamic finance widely used in banking-financial practice and considered by Islamic legal schools to be totally in conformity with the Word of Allah. They are therefore instruments of comparison particularly interesting as well as useful for the Italian legal system. They can represent a flywheel for the integration of Muslims in the social fabric, and at the same time, an antidote to the unstable European economic panorama.
\end{abstract}

SOMMARIO: 1. Premessa: 1'Islam tra religione ed economia - 2. Dalla finanza al contratto islamico, tra vincolo e libertà: alla ricerca di una teoria generale dei contratti - 3. L'etica islamica dei contratti: divieto di ribà e divieto di ghàrar - 4. Operatività delle attività della banca islamica e contrattualistica commerciale Shari'ah Compliant - 5. I contratti di tipo partecipativo: "mudàraba" e "mushàraka" - 6. (segue) Tipologie di mudàraba - 7. Cenni conclusivi e prospettive future. 


\section{1 - Premessa: 1'Islam tra religione ed economia}

L'Islam, oggi, costituisce una significativa presenza religiosa: una realtà che non può essere considerata marginale. La crescente presenza di diverse culture, tradizioni, lingue e religioni nello stesso spazio-tempo ha condotto inevitabilmente a un mutamento delle società europee che, da un punto di vista religioso, erano ancorate alle religioni cristiane.

Un recente studio condotto dal Pew Research Center ${ }^{1}$ rileva che la geografia delle religioni è continuamente destinata a cambiare. La religione cristiana nel mondo rimarrà, nei prossimi quattro decenni, molto diffusa, ma le dimensioni della comunità islamica, Ummah², aumenteranno sempre di più fino a rappresentare la comunità religiosa maggiormente in crescita. Entro il 2050, il numero dei fedeli musulmani eguaglierà il numero dei cristiani. In Italia, a causa anche dei flussi migratori, la presenza dei fedeli di religione islamica è stimata in 1.600 .000 , circa il $2,5 \%$ della popolazione ${ }^{3}$.

Indubbiamente $\mathrm{i}$ fedeli di religione islamica rappresentano un segmento della compagine occidentale che solleva una serie di nodi critici circa la possibile policy da adottare per la realizzazione di un modello d'integrazione che possa essere il più possibile inclusivo. Si profilano quindi una serie di interrogativi che spaziano in diversi ambiti, non da ultimo, nel settore giuridico-economico. È proprio quest'ultimo profilo che rimarca la necessità di indagare sui principi che stanno alla base della finanza islamica. Questa, infatti, sta diventando un importante modello alternativo di intermediazione finanziaria che, inevitabilmente, ha attirato l'interesse di istituzioni e mercati dei Paesi occidentali, motivo per cui (anche) l'Italia non può più ignorare questo fenomeno ${ }^{4}$.

* Contributo sottoposto a valutazione.

${ }^{1}$ PEW RESEARCH CENTER, The future of world religions: population growth projections, 2010-2050. Lo studio è consultabile nella sezione Publications del sito https://www.pewresearch.org/.

${ }^{2} \mathrm{Nel}$ presente contributo si è adottata una traslitterazione semplificata delle parole in lingua araba. Quindi, sono stati omessi la 'ayn, la hamza e la ta-marbuta.

${ }^{3}$ Per una mappatura degli stranieri residenti in Italia per appartenenza religiosa si rinvia allo studio condotto dalla FONDAZIONE ISMU, Immigrati e religioni in Italia (consultabile al sito https://www.ismu.org/immigrati-e-religioni-in-italia).

${ }^{4}$ In Italia, l'attenzione della dottrina (e non solo) nei confronti della dimensione islamica è via via aumentata. Nonostante ciò, non vi è mai stata una proposta sistematica volta a dare spazio all'ingresso della finanza islamica nel nostro Paese. Le ragioni, già 
La sfera contrattuale, anche quella finanziaria, nel mondo islamico è disciplinata dal diritto religioso e dall'elaborazione dottrinale dei dotti. Nonostante questa sia una dimensione profondamente tecnica, in essa si intravede in maniera ancor più nitida il paradigma etico-morale ${ }^{5}$ che pervade tutti gli ambiti del diritto islamico.

René David aveva infatti definito la religione islamica quale "la religione della legge"6, un sistema complesso - seppur eterogeneo ${ }^{7}$ - di prescrizioni che regolamentano in modo cogente la vita del fedele.

L'Islam si espande non solo secondo un ordine sociale, ma anche secondo un ordine giuridico, politico ed economico.

sollevate da una parte autorevole della dottrina, si muovono su due piani di senso: il primo è da rinvenire in un legislatore dormiente; il secondo è la scarsa pressione esercitata dalle comunità islamiche presenti in Italia. Quindi, sembra primaria la necessità di un dialogo tra Islam italiano e autorità in modo da trovare una strada di integrazione finanziaria conforme al dettato del credo religioso di questa comunità all'interno del quadro legislativo dell'ordinamento italiano. Sul punto, si rinvia a F. MIGLIETTA, P.P. RAMPINO, Gli strumenti di finanza islamica a sostegno del sistema Italia, Pearson, MilanoTorino, 2017, p. 68 ss.

${ }^{5}$ Il paradigma etico-morale richiama il concetto di giustizia sociale. Questa è un elemento ricorrente in tutto il diritto islamico e, quindi, in ogni agire umano. Esso si intravede ancora di più nell'ambito economico e in quello contrattuale, ove l'esigenza di tutela delle parti e l'equità nei rapporti tra consociati è base dell'intero impianto normativo. In altre parole, l'Islam, al vertice del sistema valoriale, pone il bene comune della Ummah: ne consegue una stigmatizzazione di tutte le azioni che vi attentino. Si pensi, in materia contrattuale, all'indulgenza del creditore nei confronti del debitore in buona fede oppure al divieto di ribà in materia di prestito di danaro. Più in generale, qualsiasi azione diretta a un mero interesse egoistico-utilitaristico da parte di un fedele viene considerata una condotta che confluisce nelle azioni considerate haram, id est condotte illecite. Cfr. V.M. DONINI, Cenni sul diritto islamico dei contratti, in Iura Orientalia VI, 2010 (www.iuraorientalia.net), pp. 260-282, in particolare, p. 276-280. Inoltre, cfr. H. BENMANSOUR, L'économie musulmane et la justice sociale, Dialogues Editions, Parigi, 1990; M. CAMPANINI, Il Corano e la sua interpretazione, Laterza, Roma-Bari, 2019, p. 117.

Sul divieto di applicazione del tasso di interesse (in arabo ribà), in cui si comprende il concetto di giustizia sociale declinato all'ambito economico, si veda anche R. HAMAURI, M. MAURI, Economia e finanza islamica. Quando i mercati incontrano il mondo del Profeta, il Mulino, Bologna, 2009, p. 21 ss.

${ }^{6}$ R. DAVID, C. JAUFFRET SPINOSI, I grandi sistemi giuridici contemporanei, $4^{\text {a }}$ edizione italiana a cura di R. SACCO, Cedam, Padova, 1994.

7 L'Islam appartiene al ceppo delle religioni abramitiche, come l'ebraismo e il cristianesimo. Ė una religione rivelata e fonda le sue radici nel Corano, il "Libro" sacro che contiene norme, appunto, eterogenee. Per comprendere tale carattere del testo coranico si rinvia a P. BRANCA Il testo sacro dei musulmani. Piccola antologia coranica, Carocci editore, Roma, 2019. 
Come è noto, il 'buon' musulmano è tenuto al rispetto della Shari' $a h^{8}$ nei molteplici aspetti della vita ${ }^{9}$, i quali sono considerati interamente sottoposti alla volontà di Allah ${ }^{10}$.

L'Islam, religione che, alla stregua dell'ebraismo e del cristianesimo, si basa sulla Rivelazione fatta da Dio ${ }^{11}$ all'uomo, è fondato sul Corano ${ }^{12}$, testo sacro rivelato da Allah, e sull'insegnamento del Profeta Muhammad. Ponendosi, quindi, come la terza religione profetica in ordine di tempo ${ }^{13}$, tra le religioni del ceppo abramitico, l'Islam è quella che più si caratterizza per una stretta relazione tra la dimensione teologico-normativa e quella sociopolitica. Il fedele-musulmano è quindi tenuto al rispetto di

8 Il termine Shari'ah letteralmente "strada battuta" "il cammino che conduce alla fonte a cui abbeverarsi", è il complesso di regole di vita e di comportamento dettato da Dio per la condotta morale, religiosa e giuridica dei suoi fedeli, e che indica la strada per la salvezza che deve essere percorsa dal fedele per raggiungere l'accesso sicuro al mondo dell'aldilà. Questi concetti sono rinvenibili nel Corano e nella Sunnah, ossia i detti, le azioni talvolta i silenzi del Profeta Muhammad, trasmessi in hadith (racconti dei compagni del Profeta). La Shari'ah si completa, infine, con la giurisprudenza islamica (figh), basata sul processo di interpretazione (igtihad) che gli esperti applicano in caso di regole non chiare, utilizzando il ragionamento deduttivo-analogico (qiyas) o fondandosi sul consenso degli esperti (igma). Le fonti qui riportate non sono tutte riconosciute dalle scuole giuridiche islamiche. Cfr., sul punto, R. HAMAURI, M. MAURI, Economia $e$ finanza islamica, cit., p. 18. Inoltre, S. FERRARI (a cura di), Introduzione al diritto comparato delle religioni. Ebraismo, islam e induismo, il Mulino Bologna, 2008, p. 191 ss.; F. CASTRO, Il modello islamico, Giappichelli, Torino, 2007, p. 12 ss.; F. CASTRO, sub voce Diritto Musulmano, in Digesto IV, vol. VI, e A. D'EMILIA, sub voce Figh (Iurisprudentia Islamica) e sub voce Shari'ah, in Novissimo Digesto italiano [Utet, Torino, 1957], ora anche in ID., Scritti di diritto islamico, raccolti (a cura di) F. CASTRO, Roma, 1976, p. 45 ss.

9 Infatti, " 1 'Islam è eminentemente un'ortoprassi". Citazione tratta da M. CAMPANINI, Il Corano e la sua interpretazione, cit., p. 9.

${ }^{10}$ In materia di teocentrismo nell'Islam, si rinvia a P. BRANCA Il testo sacro, cit., p. 17 ss.

11 Sulla concezione di Dio nella religione islamica si rinvia a M. CAMPANINI, Il Corano e la sua interpretazione, cit., p. 39 ss.

12 D'ora in avanti, citando il Corano, si farà riferimento al testo di A. BAUSANI (a cura di), Il Corano, Milano, Bur Rizzoli, 1980. Invece, su composizione e struttura del Corano si rinvia a M. CAMPANINI, Il Corano e la sua interpretazione, cit., pp. 14-36.

${ }^{13}$ Nella concezione islamica, Allah ha inviato numerosi profeti al fine di indirizzare gli uomini verso la strada della salvezza. L'Islam riconosce come profeti Adamo, Abramo, Mosè e Gesù. Maometto, con la sua predicazione, è l'ultimo profeta che completa in modo definitivo la Rivelazione divina. È questa la ragione per cui l'Islam non disconosce le altre religioni monoteistiche ma ritiene che la Rivelazione per bocca del Profeta Maometto sia quella compiuta. Cfr. M. CAMPANINI, Il Corano e la sua interpretazione, cit., p. 59-65; R. HAMAURI, M. MAURI, Economia e finanza islamica, cit., p. 15. 
determinati precetti che spaziano dalla sfera pubblica a quella privata: nessuna distinzione vi è in questo senso. La dottrina maggioritaria ${ }^{14}$ è concorde nel ritenere l'Islam non già una mera professione di fede, ma una vera e propria ideologia ${ }^{15}$ che abbraccia tanto gli aspetti legati al culto tanto quelli della vita sociale del fedele. È una realtà di appartenenza religiosa onnicomprensiva in cui esiste un fil rouge, la Shari'ah, tra i diversi spazi in cui si articola la vita del musulmano ${ }^{16}$.

Già Massimo Campanini ha evidenziato come l'Islam non sia una "religione" nell'accezione occidentale ${ }^{17}$. Il Corano, fonte primaria di risposte tanto per la sfera spirituale quanto per quella temporale, definisce l'Islam come din 18 , ossia "una concezione del mondo onnicomprensiva in cui ha un ruolo determinante la dimensione del sacro"19.

Il Libro cristallizza quindi una serie di comportamenti che il fedele deve osservare e che si estendono ben al di là della mera sfera spirituale, regolando non solo la sfera contrattuale ma anche quella civile e sociale. Difatti, nel testo sacro, vi sono molteplici ayat - versetti - dedicati a tematiche non sacre, almeno a un primo esame. Un esempio in questo senso è rappresentato dai principi in materia economica che, come si vedrà in seguito, influenzano la concezione dell'homo oeconomicus musulmano ${ }^{20}$.

Con queste premesse, risulta evidente come la materia dell'economia e della finanza, e quella che tocca i principi e le forme dei contratti presenti molti profili problematici.

${ }^{14}$ F. ROSSI-LANDI, Ideologia, Isedi, Milano, 1978, p. 5. Tra le varie, si rinvia anche a La giustizia sociale nell'Islam, tradotta in W. SHEPARD, Sayyid Qutb and Islamic Activism. A translation and Critical Analysis of "Social Justice in Islam", Brill, Leiden, p.33.; AL NAWAWI, Quaranta hadith, Cesi, Roma, 1982, p. 36.

15 Il termine ideologia è qui da intendersi "nel senso positivo del termine, cioè come filosofia e concezione globale della realtà. È vero che, spesso, si inclina a considerare l'ideologia come alienazione, falsa coscienza, manipolazione delle menti. Ma si è evidenziato come l'ideologia sia anche azione e progettazione sociale". Cfr. M. CAMPANINI, Il Corano e la sua interpretazione, cit., p. 6 s.

16 S. FERRARI (a cura di), Introduzione al diritto comparato delle religioni, cit., p. $191 \mathrm{ss.}$

17 M. CAMPANINI, Il Corano e la sua interpretazione, cit., p. 3 ss.

18 Il Corano, V:3 e III:19. Il termine din significa: iman, ihsan,'islam, id est., rispettivamente, fede, retto comportamento e sottomissione. Cfr. M. CAMPANINI, Il Corano e la sua interpretazione, cit., p. 3 s.

${ }^{19}$ M. CAMPANINI, K. MEZRAN, Arcipelago Islam, Laterza, Roma-Bari, 2007, p. IX.

${ }^{20}$ R. HAMAURI, M. MAURI, Economia e finanza islamica, cit., p. 18 ss. 
In primo luogo, il giurista occidentale che si interfaccia con il diritto islamico dei contratti si trova dinanzi ad alcune difficoltà ${ }^{21}$ da tenere in considerazione.

La prima riguarda la terminologia: il diritto islamico si sviluppa in contesti in cui la lingua di riferimento è l'arabo. Studiare un diritto come quello islamico richiama gli studiosi a dovute precauzioni e attenzioni dal punto di vista terminologico, nonché semantico. Tradurre letteralmente un termine non è garanzia di corrispondenza con le categorie giuridiche occidentali. Quindi, al di là della mera traduzione letterale, è necessario ricercare lo stesso concetto semantico nella tradizione giuridica occidentale.

In secondo luogo, va tenuto in considerazione la profonda religiosità del mondo giuridico islamico: il diritto islamico classico è l'unico diritto e sarebbe impossibile che una branca del diritto, anche in senso contemporaneo, avesse vita senza alcun possibile riferimento ai principii religiosi insiti nella Rivelazione.

In aggiunta a questa difficoltà, vi è un diverso approccio metodologico dei giuristi musulmani alla materia contrattuale: nel diritto islamico manca una teoria generale dei contratti. Il modus agendi del giurista musulmano in questo settore muove dallo studio delle fattispecie particolari per poi, secondariamente ed eventualmente, cristallizzare una regola generale. I giuristi occidentali, invece, operano in maniera deduttiva, ricavando la regola applicabile alla fattispecie concreta da principi generali e astratti.

Sarà dunque necessario prendere in considerazione tutti questi aspetti, sottolineando altresì peculiarità, similitudini e profonde diversità rispetto alla legislazione contrattuale occidentale.

\section{2 - Dalla finanza al contratto islamico, tra vincolo e libertà: alla ricerca di una teoria generale dei contratti ${ }^{22}$}

$\mathrm{L}^{\prime}$ Islam ${ }^{23}$ indica chiari principi economici ai propri adepti anche in materia finanziaria ${ }^{24}$.

${ }^{21} \mathrm{Ci}$ si riferisce alle difficoltà insite nel metodo della comparazione giuridica come già evidenziato da R. SACCO, P. ROSSI, Introduzione al diritto comparato, Utet, Milano, 2019, pp. 1-184.

22 Per maggiori approfondimenti D. SANTILLANA, Istituzioni di diritto musulmano malichita, con riguardo anche al sistema sciafiita, Istituto per Oriente, Roma, 1938. 
Esaminare i precetti che regolano la finanza islamica vuol dire richiamare l'insieme di transazioni, contratti finanziari e istituzioni bancarie fondate sui precetti della Shari'ah. In altri termini, un'economia è islamica solo se i mercati e gli strumenti in essa utilizzati sono considerati halal $^{25}$, quindi leciti e conformi alla Shari'ah.

L'Islam sposa una filosofia economica che mira a incentivare il fedele al lavoro, intellettuale o manuale che sia, come strumento di emancipazione sociale. L'ottenimento di proventi dallo sforzo lavorativo è considerato un comportamento halal a opera dei componenti della Ummah. Il guadagno economico e il profitto personale sono ben accetti dalla religione islamica.

Come in tutte le strutture sociali, però, le tensioni economiche causate dalla povertà possono sfociare in redditi derivanti da comportamenti amorali come furto, frode, inganno e simili. Il sistema coranico, al fine di prevenire tali situazioni, offre alcune prescrizioni che mirano a ridurre le differenze economico-sociali attraverso dei meccanismi creati ad hoc. In tal senso, si pensi all'elemosina obbligatoria - la zakat ${ }^{26}$ che da un lato permette la ridistribuzione del reddito in eccesso e dall'altro ne consente la purificazione.

L'Islam si prende cura degli interessi individuali senza che questi nuocciano alla collettività; inoltre, esso cura i fabbisogni primari dei ceti meno abbienti per evitare che questi percorrano strade illecite. In breve, la povertà è ritenuta dall'Islam una minaccia all'esistenza stessa della fides. Dunque, il modello economico islamico è ispirato al principio secondo il quale tutti i membri della comunità islamica devono riuscire a ottenere una soglia minima di reddito sufficiente per vivere ${ }^{27}$.

$23 \mathrm{Si}$ fa riferimento in questa sede al sistema giuridico islamico tenendo in considerazione, nella materia qui trattata, delle interpretazioni dottrinali in materia economica (e poi finanziaria) e anche contrattuali.

24 D. ATZORI, Fede e mercato: verso una via islamica al capitalismo?, il Mulino, Bologna, 2010, p. 41 ss.

25 Nell'Islam, halal (lecito) indica quanto è permesso in ogni ambito della vita, ed è contrapposto a haram (proibito). Cfr. F. CASTRO, Il modello islamico, cit., 2007, p. 12 ss.; S. FERRARI (a cura di), Introduzione al diritto comparato delle religioni, cit., p. $197 \mathrm{~s}$.

${ }^{26}$ R. HAMAURI, M. MAURI, Economia e finanza islamica, cit., p. 26 ss.; D. ATZORI, Fede e mercato, cit., p. 21 ss.; G. VERCELLIN, Istituzioni del mondo musulmano, Einaudi, Torino, 1996, p. $220 \mathrm{~s}$.

27 Sul sistema della zakat come meccanismo per l'eliminazione della povertà mi permetto di rinviare a H. AHMED, Role of Zakah and Awqaf in Poverty Alleviation, Islamic Research and Training Institute, Jeddah, 2004. 
Da ciò si deduce che i principi che regolano il sistema finanziario islamico non esulano dall'ambito religioso dell'agire umano, bensì ne rappresentano un modus operativo che realizza appieno il Logos di Allah ${ }^{28}$. Il testo sacro dell'Islam, il Corano, offre prescrizioni precise su alcuni profili della vita economica dei fedeli. Questo è uno dei primi punti di differenza con la visione economica occidentale.

In Occidente, l'economia guarda agli aspetti strettamente materiali dell'uomo, invece, la religione, il lato più intimo, spirituale. Queste due realtà, nel mondo islamico sono sovrapposte in un più ampio disegno che include utilità e aldilà ${ }^{2}$. In specie, l'economia è uno dei molteplici settori in cui i musulmani realizzano il disegno salvifico tracciato da Dio per i fedeli.

L'Islam sancisce la libertà nell'attività negoziale e imprenditoriale ${ }^{30}$ e la possibilità di fare accordi ${ }^{31}$, ma pone al tempo stesso argini a tali libertà. In altre parole, promuove l'iniziativa economica e la libertà negoziale cristallizzando in modo chiaro i confini di tale agire.

I precetti che si riferiscono al diritto dei contratti islamici trovano fonte primaria nel Corano. Nella versione di 'Othman ${ }^{32}$, è un corpus ben strutturato di precetti idonei a dettare regole comportamentali ai fedeli. Il "Libro", però, non è una codificazione normativa nell'accezione secolare; è un testo che è considerato dalla religione islamica completo - oltre che inimitabile e autentico - e offre tutte le risposte per il fine che si prefigge, difettando di quella sistematicità così come intesa nell'ottica giuridica occidentale $^{33}$. Si tratta di un testo ricco di aspetti narrativi, simbolici,

${ }^{28}$ Cfr. M. d'ARIENZO, I fondamenti religiosi della finanza islamica, in Stato, Chiese e pluralismo confessionale, Rivista telematica, (https://www.statoechiese.it), luglio 2012, pp. 19.

${ }^{29}$ R. HAMAURI, M. MAURI, Economia e finanza islamica, cit., p. 59 ss.

30 Il Corano, II:275.

31 Il Corano, IV:29.

32 In materia di composizione e struttura del Corano si veda M. CAMPANINI, Il Corano e la sua interpretazione, Roma-Bari, Laterza, 2019, p. 14 ss. L'Autore mette in evidenza le tappe storiche che hanno condotto alla stesura del testo sacro. Il terzo califfo, 'Othman (644-656), istituì una commissione che pose fine definitivamente ai dibattiti sulla lunghezza e sull'ordine dei capitoli del Corano. Nella sua versione consolidata, il Libro è strutturato in 114 sure (capitoli) a loro volta divise in ayat (versetti).

33 L'orientalistica ha posto l'accento sul tema e si è molto discusso fino a che punto il Corano possa essere ritenuto un codice. Cfr., sul punto, M. CAMPANINI, Il Corano e la sua interpretazione, cit., p. 55 ss. Inoltre, si veda anche S. BELAID, Islam et droit, Centre de Publications Universitaire, Tunis, 2000, p. 284. 
omiletici, escatologici. Solo una parte relativamente ristretta dei versetti circa un decimo del totale - è ritenuta dalle scuole giuridiche musulmane avere carattere giuridico ${ }^{34}$ stricto sensu.

È indubbio che il Corano prenda in esame, disciplinandoli, i rapporti tra l'uomo e suoi simili. Questi vengono intesi come muamalat ${ }^{35}$, rapporti giuridici tra consociati che si sviluppano dal diritto di famiglia al diritto commerciale, dal diritto ereditario a quello penale. Ne consegue che il contratto è un istituto giuridico muamalat che si concretizza in una dimensione orizzontale, ossia man to man.

Il Corano, tuttavia, manca di una disciplina dettagliata in materia contrattuale, volendo essere una prassi comportamentale 'coranicamente' orientata più che una codificazione giuridica ${ }^{36}$.

Già il termine contratto comunemente inteso dai giuristi islamici ha una più ampia portata rispetto a quella usata dai giuristi italiani e, più in generale, dai giuristi di tradizione romanistica, per i quali il contratto è un atto (almeno) bilaterale attraverso cui è possibile creare, regolare o estinguere un rapporto giuridico di natura economica ${ }^{37}$.

Nel diritto islamico la situazione risulta essere differente. La terminologia del contratto islamico ${ }^{38}$ presenta criticità semantiche dal momento che si è soliti tradurre il termine 'aqd ${ }^{39}$ con il termine vincolo che giuridicamente si presta a diverse possibili interpretazioni: 'aqd deriva dal verbo aqada, letteralmente "annodare", e viene impiegato, da un punto di vista concettuale, per tutte le obbligazioni derivanti dalla manifestazione

34 P. BRANCA $I l$ testo sacro, cit., p. 95.

35 Il diritto islamico conosce, nei rami del diritto, regole di culto, le ibadat, e regole sociali dette, appunto, muamalat. Le prime riguardano la relazione che intercorre tra l'uomo e Dio (ad esempio i cinque pilastri dell'Islam); le seconde, i rapporti tra i consociati. In quest'ultima categoria rientrano il matrimonio, i diritti reali, le obbligazioni e il diritto successorio. Cfr., sul punto, F. CASTRO, Il modello islamico, cit., 2007, p. 24.; S. FERRARI (a cura di), Introduzione al diritto comparato delle religioni, cit., p. 192.

${ }^{36}$ Cfr. S. BELAID, Islam et droit, cit., p. 284.

37 Per un'analisi del diffuso dibattito dottrinale in materia di contratto nell'ordinamento italiano si veda, a mero titolo esemplificativo, R. SACCO, G. DE NOVA, Il contratto, in Trattato di diritto privato diretto da P. RESCIGNO, $4^{\mathrm{a}}$ ed., vol. X, Utet, Torino, 2018. Invece, per una ricostruzione della teoria del contratto italiano si rimanda a L. BALESTRA, Introduzione al diritto dei contratti, il Mulino, Bologna, 2015.

38 Il contratto è considerato dalle scuole giuridiche islamiche al-'Aqd shari'at almuta'aqidayn, id est "Shari'ah tra i consociati"; sul punto V. DONINI, Cenni sul diritto islamico, cit., p. 273.

39 Sul punto si consulti F. CASTRO, Il modello islamico, cit., 2007, p. 69 s. 
di volontà di un soggetto ${ }^{40}$. Ne consegue che 'aqd viene utilizzato per una categoria di atti più estesa rispetto a quella rientrante nella nozione di contratto occidentale, ad esempio viene utilizzato anche per atti unilaterali, o meri negozi giuridici come il matrimonio ${ }^{41}$.

Massima attenzione, quindi, deve essere posta all'uso del termine 'aqd nel diritto islamico dei contratti. Non sono mancate voci, tra i giuristi islamici ${ }^{42}$, che volessero usare il termine in questione solo per i contratti intesi in senso occidentale. In altri termini, essi vorrebbero impiegare 'aqd in una versione restricta, orientata dalla cultura giuridica del vecchio continente; questo come risultato dei fenomeni di colonizzazione che hanno di fatto condizionato la cultura giuridica islamica.

Volendo comunque ricercare regole volte alla ricostruzione di una teoria generale del contratto, è necessario fare un lavoro di rammendo tra $\mathrm{i}$ vari nuclei di portata generale del tessuto del dettato shariatico, i cosiddetti qawa'id kulliyya ${ }^{43}$.

Primo assioma è quello della forza del vincolo contrattuale tra le parti, principio comune a tutti i sistemi giuridici ${ }^{44}$. Il Corano cristallizza indubbiamente questo profilo nella sua lettera: "O voi che credete, adempiete ai patti"45. Vi è, tuttavia, un ayat dalla portata più precisa che rivela la presenza di Allah a garanzia del vincolo: "E non rompete i giuramenti che avete solennemente pattuito, poiché è Dio che avete costituito sopra di voi a garante, e Dio sa ciò che fate" 46 . Ne risulta, dunque, la concezione secondo

${ }^{40}$ Cfr., sul punto, V. DONINI, Cenni sul diritto islamico, cit., p. 260 ss.

41 Nell'ottica di un giurista occidentale è impensabile poter impiegare il concetto di contratto all'istituto del matrimonio, il quale viene considerato un negozio giuridico che non persegue finalità esclusivamente connesse alla sfera economica. Al contrario, nel diritto islamico, il matrimonio è un contratto consensuale tra uomo e donna. Per un'analisi completa dell'istituto si veda S. FERRARI (a cura di), Introduzione al diritto comparato delle religioni, cit., p. 205 ss. Inoltre, F. CASTRO, Il modello islamico, cit., 2007, p. 40 ss.

42 Per una visione d'insieme, tra le correnti di giuristi più conservatrici e quelle più moderniste, si rimandar a M. PAPA, La definizione di contratto e l'autonomia contrattuale in diritto musulmano: dai principi della Shari'ah alle legislazioni contemporanee, in Roma e America Latina, 1999, p. 285.

43 Traducibile semanticamente (dall'arabo) con "principi generali".

${ }^{44}$ Questo è un profilo presente già nel diritto romano dei contratti, il quale è espresso con il brocardo latino "pacta sunt servanda". Cfr. V. ARANGIO-RUIZ, Istituzioni di Diritto Romano, 14a ed., Jovene, Napoli, 1984.

45 Il Corano, V:1.

46 Il Corano, XVI:91. Si prenda in esame anche Il Corano, II 177. 
cui Dio è testimone dei rapporti tra fedeli, quasi una figura antropomorfica del divino che presenzia alla genesi del contratto ${ }^{47}$. $\grave{E}$ in forza di questa presenza che il fedele si atterrà al rispetto dei contenuti del patto e non tanto e non soltanto per un generico atteggiamento riconducibile alla buona fede contrattuale quale configurata dalla contrattualistica occidentale.

Nel diritto islamico dei contratti la forza vincolante, al di là del rispetto della parola data e della certezza dei rapporti economico-sociali, ha perciò un aspetto marcatamente religioso. Emerge qui la funzione pedagogica $^{48}$ della religione islamica, che tende a orientare i comportamenti dei fedeli. Il buon musulmano, essendo sottomesso alla volontà divina, per non porsi in una situazione di violazione della legge, tenderà a rispettare quanto più possibile ogni prescrizione. In particolare, con riferimento alla materia trattata, il fedele rispetterà il vincolo contrattuale come conseguenza di un valore religioso a cui ritiene di dover ottemperare, in quanto contenuto della Rivelazione. Quindi, il principio della forza vincolante ha carattere assoluto e non è possibile abrogarlo in quanto applicabile a qualsiasi forma di accordo e di contratto.

Altro assioma con valenza generale del diritto islamico dei contratti è quello della libertà contrattuale ${ }^{49}$.

47 V. DONINI, Cenni sul diritto islamico, cit, p. 272.

48 La regione in cui l'Islam si è sviluppato è nota come Higaz, costa occidentale dell'Arabia. Prima della rivelazione di Maometto, le tribù preislamiche erano politeiste, con rituali alquanto primitivi come i riti sacrificali, venerazioni di pietre, figure sacerdotali assimilabili a uomini-indovini, etc. Con l'avvento dell'apostolato del Profeta, al di là dei profili strettamente legati al culto, si può evidenziare una discontinuità rispetto al precedente periodo. Ne consegue che l'Islam ha educato i costumi delle genti dell'epoca svolgendo di fatto un ruolo pedagogico. Anche se si prenderanno in esame più avanti gli aspetti legati al commercio, sin d'ora è possibile affermare che i commercianti di fede musulmana prediligevano intrattenere affari con altri commercianti di fede musulmana perché rassicurati dalla condivisione del medesimo sistema etico. In breve, la religione come maestra della condotta umana su cui si rinvia a F. CASTRO, Il modello islamico, cit., 2007, p. 40 ss.

49 Inizialmente questo profilo non fu preso minimamente in considerazione; anzi, le scuole giuridiche islamiche seguivano un metodo più rigido, ritenendo contratti halal solo quelli tipizzati dalla Rivelazione a pena di nullità; i contratti, quindi, erano un numero chiuso.

Solo grazie alla scuola giuridica hanbalita si è introdotto il principio della libertà contrattuale, pur sempre con divieti da rispettare nell'agire contrattuale. Attraverso un ragionamento analogico, gli hanbaliti hanno enucleato questo principio che ha una rilevanza notevole per la contrattualistica contemporanea, soprattutto in materia di contratti finanziari (vedi infra). Sul punto, cfr. V. DONINI, Cenni sul diritto islamico, cit., p. 
Tale libertà è un precipitato della teoria della tolleranza, 'ibaha, a immagine della libertà concessa all'uomo nel momento della creazione nel rispetto della legge di Dio ${ }^{50}$. Ne consegue che i fedeli musulmani godono della libertà contrattuale purché si rispetti la Shari'ah; laddove si rispetti tale condizione, il contratto ha forza vincolante tra le parti.

A differenza del principio della forza vincolante, quello della libertà contrattuale però non ha portata incondizionata, essendo mitigato dal paradigma della giustizia sociale ${ }^{51}$. Su questo punto, un ruolo fondamentale è quello del giudice, il quale è chiamato a riequilibrare l'assetto tra le parti ove vi sia uno squilibrio contrattuale che possa minare il paradigma della giustizia sociale. Quindi, la libertà contrattuale è fortemente limitata dalla morale islamica, con il potere del giudice di riequilibrare l'obbligazione laddove questa risulti socialmente ingiusta. $\mathrm{Ne}$ segue che il principio dell'equità - istihsan - rappresenta un altro elemento rientrante nella teoria generale del contratto islamico.

L'istihsan è un parametro che guida il giudice quando questi debba utilizzare il ragionamento analogico per supplire all'assenza di una regola puntuale per regolare una fattispecie concreta. Oltre a ciò, è prezioso strumento per dare risposte agli inevitabili mutamenti storico-sociali cui anche l'Islam è chiamato a dare risposte nonostante la portata relativamente immutabile del suo diritto.

L'equità islamica ${ }^{52}$ assicura la capacità di adattamento del diritto al caso concreto potendo il giudice financo disapplicare il precetto religioso

273 s. Per un approfondimento in materia di libertà contrattuale, si rinvia a D. SANTILLANA, Istituzioni di diritto musulmano malichita, cit., p. 83.

50 "Ogni contratto tra i musulmani è valido, tranne quello che dichiari proibito ciò che è permesso, e permesso ciò che è proibito", hadith citato in V. DONINI, Cenni sul diritto islamico, cit., p. 274.

51 Proprio partendo dal concetto di giustizia sociale, la finanza islamica è cresciuta negli ultimi anni, attecchendo anche in Occidente: essa è infatti tema attualmente centrale nell'ottica di una più ampia tutela delle parti considerate deboli contrattualmente, divenendo principio che sta contribuendo alla creazione di nuovi prodotti finanziari etici nel sistema bancario convenzionale danneggiato dagli ultimi episodi legati alla crisi finanziaria del 2008 e diretto verso l'apertura a sistemi finanziari alternativi. Su questo versante, si rinvia al testo di T.V. RUSSO, I contratti Shari'ah compliant. Valori religiosi e meritevolezza degli interessi, Edizioni scientifiche italiane, Napoli, 2014, p. 61 ss., il quale conduce un'analisi puntuale sulle forme di contratto impiegate dalle banche islamiche ove emerge l'etica sociale islamica.

52 Anche altri diritti religiosi possiedono all'interno del proprio ordinamento giuridico uno strumento analogo all'equità islamica. Basti pensare all'aequitas prevista dall'ordinamento canonico che funge da strumento elastico seppure con un fine diverso. 
al caso particolare quando la sua applicazione comporterebbe un danno al fedele per assicurare la giustizia sociale. Tale principio, pur muovendosi su un binario processuale, riverbera effetti sulla realtà sostanziale, incidendo così sul diritto contrattuale. È un principio, quello dell'equità, che non è solo applicabile al diritto dei contratti, ma è in questo settore che ha una serie di conseguenze importanti che spaziano dall'equilibrio del sinallagma contrattuale, alla ricerca di una giustizia sociale, all'indulgenza nei confronti del debitore inadempiente ma in buona fede, nonché al divieto di arricchimento ingiustificato.

Come detto, il principio di equità è applicato dal giudice in un momento ex post, i.e. quando vi è ingiustizia nel contratto. Da ciò, discende la proibizione di qualsiasi arricchimento ingiustificato a opera di una parte. È un corollario che, al di là delle diverse scuole giuridiche, ha valenza universale. Quello dell'istihsan limita il campo di azione degli altri principi generali della forza vincolante, da un lato, e della libertà contrattuale, dall'altro.

\section{3 - L'etica islamica dei contratti: divieto di ribà e divieto di ghàrar}

Il principio di equità si manifesta più massicciamente nella contrattualistica finanziario-bancaria. Il principale corollario economicofinanziario è il divieto di applicazione dell'interesse ${ }^{53}$, noto come divieto di ribă ${ }^{54}$. Il Corano più volte cita questa proibizione senza definirne la

Infatti, l'equità canonica è volta ad assicurare la salvezza dell'anima (cfr. can. 1752) dei fedeli e solo mediatamente fini di giustizia collettiva, mentre l'equità islamica funge da mezzo per garantire primariamente la giustizia sociale con una più spiccata attenzione alla dimensione comunitaria. In materia di equità canonica si rimanda a B. SERRA, Sull'equità canonica quale oggetto di una pretesa giuridicamente esigibile, in Stato, Chiese e pluralismo confessionale, cit., n. 36 del 2017, pp. 1-18.; S. BERLINGÒ, Suppletio legis, in Stato, Chiese e pluralismo confessionale, cit., settembre 2009, p. 3 ss.

53 Il tasso di interesse è il costo del credito o il rendimento del risparmio. Cfr., sul punto, G. ANTONELLI, N. DE LISO, G. GUIDETTI, R. LEONCINI, G. VITTUCCI MARZETTI, L. ZAMPARINI, Economia, Giappichelli, Torino, 2019, p. 143 ss.

54 Il termine arabo ribà, dalla radice $r b w$, ha il senso letterale di "aumento", "accrescimento" infatti "Quel che voi prestate a usura [ribà] perché aumenti sui beni degli altri, non aumenterà presso Dio" (Il Corano, XXXX:39). Si può notare come nella seconda parte del versetto in esame il verbo rabà-yarbu è usato con l'accezione "aumenterà" i meriti di colui che sarà generoso verso i poveri. Quando, ribà, lo si riferisce alle obbligazioni (pecuniarie) è reso con il termine usura sensu lato. Cfr. Encyclopédie de l'Islam. Nouvelle èdition, E.J. Brill, Leiden, 1995, p. 508. G.M. PICCINELLI, Banche islamiche in 
portata semantica. Ad esempio: "O voi che credete! Non praticate il ribà, doppiando o raddoppiando, e temete Dio sì che possiate essere felici" 55 , ancora, "Ma Dio ha permesso la compravendita e ha proibito il ribà [...]"56.

Alla luce dei succitati versetti, gli studiosi del diritto islamico ${ }^{57}$ hanno preso in esame questo divieto più volte, addivenendo in un primo momento a una interpretazione orientata a vietare la pratica dell'usura ${ }^{58}$, e quindi a rendere ammissibile l'interesse entro un certo tasso. Successivamente, l'orientamento è mutato in un divieto di applicazione di qualsiasi forma di surplus in danaro indipendente dal raggiungimento di uno scopo. In sostanza, si è vietato, ex ante, un prezzo sul danaro dato in prestito monetizzando, così, il tempo del pagamento differito del debitore. Dunque, i giuristi e gli economisti islamici riconoscono, parimenti al mondo occidentale, la moneta come mezzo di scambio e strumento di conto, ma convergono nel ritenere che la Shari'ah espunga la concezione di riserva di valore della moneta. Questa non può generare ricchezza in quanto tale, bensì solo se inserita in un processo produttivo non indipendente dal risultato.

Il corollario economico del divieto di ribà vuole evitare squilibri tra le parti coinvolte in un'operazione finanziaria: il businessman, infatti, è suscettibile sia a perdite che a profitti, mentre un intermediario bancario ha solo profitti al di là dell'andamento dell'attività svolta dal primo. Ciò pone il debitore in una posizione di asimmetria rispetto al suo creditore, il quale, senza dubbio alcuno, si trova in una posizione di preminenza

contesto non islamico, Roma, IPO, 1996, p. 19 s.; G.M. PICCINELLI, Il diritto agli interessi nei paesi arabi, in Diritto del commercio internazionale, 1996, p. 35 ss.; N. SALEH, A. AJAI, Unlawful Gain and Legittimate Profit in Islamic Law: riba, gharar, and Islamic banking, 2nd ed., Graham \& Trotman, London-Boston, 1992; A. SAEED, Islamic banking and interest: A study of the Prohibition of Riba and its Contemporary Interpretation, vol. 2, Brill Academic Publishers, Leiden, 1996.; M. UMER CHAPRA, The Nature of Ribà in Islam, working paper, Hamdard Islamicus, 1984.; M. RONDISON, Islam et capitalisme, Editions du Seuil, Paris, 1996.

55 Il Corano, III:130.

${ }^{56}$ Il Corano, II 275-280.

${ }^{57} \mathrm{Si}$ rinvia per una ricostruzione dettagliata in materia di interpretazione del principio di ribà a G.M. PICCINELLI, Banche islamiche, cit., p. 22 ss.

${ }^{58}$ In materia di usura, anche se in riferimento alla Chiesa cattolica, si rinvia a $\mathbf{S}$. NOTO, Tra economia della religione ed economia religiosa. Spigolature italiane di storia e teoria economica, in Stato, Chiese e pluralismo confessionale, cit., n. 10 del 2020, p. 72 ss. 
contrattuale che, nell'ottica shariatica, contrasterebbe con l'equità e la giustizia sociale ${ }^{59}$ previsti dal disegno divino di Allah.

La finanza islamica edifica l'intera struttura basandosi su un meccanismo in cui le parti condividono profitti e perdite di qualsiasi attività finanziaria; in altre parole, le parti coinvolte raggruppano una porzione del loro capitale/lavoro finalizzato allo schema 'to share' di business. È una tecnica che incontra risultati positivi nell'economia reale e sulla ridistribuzione del reddito. In questo senso le banche, gestori di fondi, giocano un ruolo importante nell'applicazione concreta di questo postulato ${ }^{60}$.

Questa concezione delle dinamiche finanziarie ha influenzato la struttura bancaria islamica61, facendo sì che questa si contrapponga, $\mathrm{o}$ perlomeno sia alternativa, a quella cosiddetta "convenzionale", che costruisce l'intero sistema sul meccanismo del tasso di interesse ${ }^{62}$.

Il divieto di ribà è indubbiamente il principio cardine attorno al quale ruota l'intero sistema economico islamico, ma non è l'unico. Accanto a questo si trovano altre prescrizioni, come il ghàrar63 e il maysìr64. Il primo di questi termini significa rischio, incertezza e ciò vieterebbe l'introduzione di elementi imprevedibili, non chiari o comunque incompleti in un contratto, o più in generale, in un'operazione finanziaria. Ne consegue che il divieto di ghàrar vuole evitare incertezza su elementi di una transazione che possano condurre un contraente in un'alea di rischio vietando dunque situazioni di asimmetria informativa tra le parti,

${ }^{59}$ La portata pratica della giustizia sociale nell'Islam ha dei riflessi sulla concezione di distribuzione della ricchezza nel mondo islamico; sul punto si confronti R. HAMAURI, M. MAURI, Economia e finanza islamica, cit., p. 27 ss.

${ }^{60}$ R. HAMAURI, M. MAURI, Economia e finanza islamica, cit., p. $78 \mathrm{~s}$.

61 Sulla nozione di banca islamica e sul meccanismo di raccolta del risparmio, si rinvia a G.M. PICCINELLI, Banche islamiche, cit., p. 69 ss.

62 Le differenze e le similitudini che vi sono tra banca islamica e banca convenzionale sono delineate in modo esaustivo in P. BIANCONE, La banca islamica, Giappichelli, Torino, 2017, p. 69 ss.

63 Il divieto di ghàrar fonda il suo meccanismo sulla stessa logica del divieto di ribà. L'alea di rischio attenta l'equilibrio tra prestazioni reciproche, il quale è cardine del diritto islamico. È necessario però rimarcare, in questa sede, che il divieto di ghàrar non si fonda su alcun versetto coranico specifico ma solo su un generico divieto di gioco d'azzardo. In particolare, il Corano II:219: “Ti domanderanno ancora del vino e del maysir. Rispondi: c'è peccato grave e ci sono vantaggi per gli uomini in ambo le cose: ma il peccato è più grande del vantaggio". Cfr. V. DONINI, Cenni sul diritto islamico, cit., p. $281 \mathrm{~s}$.

${ }^{64}$ R. HAMAURI, M. MAURI, Economia e finanza islamica, cit., p. 79 s. 
indeterminatezza sul prezzo, sull'oggetto, sulle qualità o sulla quantità del bene $^{65}$. Il principio in esame, tuttavia, non ha carattere di assolutezza e rileva nel caso in cui l'incertezza sia rimarchevole rispetto alla natura dalla transazione.

Il maysìr, invece, è l'intento speculativo legato a un evento. Concetto, questo, che richiama in modo esplicito il gioco d'azzardo, considerato vietato dalla Legge islamica in quanto avente una finalità meramente speculativa e non di copertura dei rischi.

Talvolta, il principio di maysir viene accostato al termine qimar che ha come elemento comune col primo l'imprevedibilità di un evento. Al qimar, però, consegue necessariamente l'impoverimento di un altro soggetto mentre nella fattispecie del maysìr l'arricchimento è strettamente collegato a un evento futuro ${ }^{66}$.

In un'ottica de iure condendo, il divieto di ghàrar e quello di maysìr vietando l'aleatorietà nei rapporti economico-giuridici, pongono molteplici problemi soprattutto in materia di assicurazione (takaful) ${ }^{67}$. Settore questo in forte crescita ed espansione negli ultimi anni, sicuramente il comparto assicurativo presenta particolari problemi di compatibilità normativa rispetto alla cornice in cui si muove ${ }^{68}$.

A chiudere il novero dei pilastri islamici in materia economica vi è la proibizione del commercio o dell'investimento in beni e/o attività considerate haram, ossia proibite.

Si tratta di un corollario economico definibile come indiretto, nel senso che l'Islam è ortoprassi ${ }^{69}$ che modula ogni aspetto della vita del

65 R. LOHLKER, Shari'ah und Moderne: Diskussionen über Shwangerschaftsabbruch, Versicherung und Zinses, Steiner, Stuttgart, 1996, p. 86.

66 F. MIGLIETTA, P.P. RAMPINO, Gli strumenti di finanza islamica, cit., p. 11. Gli Autori, per meglio chiarire la differenza che intercorre tra maysir e qimar, riportano un efficace esempio che, in questa sede, può sicuramente aiutare a comprendere lo spartiacque tra i due elementi. Quindi, "vi è maysìr in una lotteria mentre vi è qimar in un gioco di carte dove la vincita di un soggetto è il contraltare del denaro perso dall'altro".

67 In materia di assicurazione islamica si rimanda a P. BIANCONE, La banca islamica, cit., p. 199 ss.; S.A. NAZIM, N. SHARIQ, Takaful and Islamic Cooperative Finance: Challenges and Opportunities, Edward Elgar, Cheltenham, 2016.

68 Per un'analisi sulle problematicità che l'assicurazione islamica incontra nel percorso di affermazione si rinvia a S.O GÖNÜLAL, Takaful and Mutual Insurance: Alternative Approaches to Managing Risks, Washington, DC, World Bank, 2013; H. SHEILA., A. MOHAMED, S. YOUNES, R.Z. HANNA, S. IBRAHIM, Accounting Auditing and Governance for Takaful Operations, Wiley, Hoboken, New Jersey, 2012.

${ }^{69}$ M. CAMPANINI, Il Corano e la sua interpretazione, cit., p. 9. 
fedele e, in alcune circostanze, vieta il consumo di determinati beni, tra cui bevande alcoliche, carne di suino, armi, tabacco, gioco d'azzardo e pornografia. Il divieto si estende anche a tutti i beni cosiddetti derivati, soprattutto nel campo alimentare come nel caso dello strutto, del caglio e delle gelatine. Queste proibizioni trovano accoglimento nelle attività di investimento, anche azionario, in imprese coinvolte nelle suddette attività $^{70}$.

Dunque, il rapporto tra il fedele musulmano e il danaro è fortemente e profondamente influenzato dalla dimensione appena descritta: il danaro per l'Islam viene considerato un mezzo non un fine ${ }^{71}$. Ciò, inevitabilmente, riverbera effetti sulla struttura degli intermediari bancari e i prodotti e/o servizi da questi offerti ${ }^{72}$.

Infatti, al di là dei meccanismi delle figure contrattuali ${ }^{73}$, un ruolo di primo piano nell'operatività dei principi dell'economia del Profeta è la costante presenza all'interno delle istituzioni finanziarie dello Shari'ah Supervisory board o, anche, Shari'ah Commitee ${ }^{74}$. È un organo fortemente raccomandato dalle best practices delle istituzioni islamiche al fine di vigilare sulla conformità shariatica dei prodotti e servizi finanziari offerti dall'intermediario.

La Shari'ah Board è organismo, stando alle esperienze attuali, composto da un numero variabile di esperti, solitamente da un minimo di tre a un massimo di sette, i quali sono chiamati a emettere dei pareri in materia di attività e prodotti finanziari assimilabili a delle vere e proprie fatàwa ${ }^{75}$. Queste sono fondamentali per l'operatività di un islamic bank, in

70 Il Corano, V:91-92.

${ }^{71}$ Questo aspetto si evince dall'intero impianto del comparto della finanza islamica, sul punto, si veda R. HAMAURI, M. MAURI, Economia e finanza islamica, cit., p. 18 ss.

72 P. BIANCONE, La banca islamica, cit., 79 ss.

${ }^{73}$ R. HAMAURI, M. MAURI, Economia e finanza islamica, cit., p. 80 ss.; in materia di compatibilità della finanza islamica alla normativa italiana si veda P. BIANCONE, S. SECINARO, M. RADWAN, Compatibilità della finanza islamica alla normativa italiana, in Stato, Chiese e pluralismo confessionale, cit., n. 9 del 2020, p 1 ss.; inoltre, S. ALVARO, La finanza islamica nel contesto giuridico ed economico italiano, in Quaderni di ricerca giuridica Consob, 6, 2014, pp. 1-68.

74 P. BIANCONE, S. SECINARO, M. RADWAN, Compatibilità della finanza islamica, cit., p. 3 s.; M. RADWAN, Adoption of Unconventional Financial Institutions: A European Perspective, Giappichelli, Torino, 2019, p. 18; R. HAMAURI, M. MAURI, Economia $e$ finanza islamica, cit., p. 98.

75 La parola significa "novità", "chiarificazione", "gioventù", "perfezione", "spiegazione", "ricerca una decisione da una corte": compare undici volte nel Corano, in cinque sure diverse. La fatwa è il risultato di due azioni: l'istifta, la domanda, e l'ifta, la 
quanto certificano i prodotti che, in caso di contrarietà ai principi islamici, non vengono offerti ai clienti. In alcune ipotesi gli esperti possono essere coadiuvati, in caso di situazione d'impasse, da consulenti esterni che esprimono pareri ad hoc in materia contrattuale, d'investimento e/o in materia bancaria.

\section{4 - Operatività delle attività della banca islamica e contrattualistica commerciale Shari'ah Compliant}

Come si è accennato nel paragrafo precedente, i principi economici dell'Islam trovano spazio nella finanza islamica ma si interfacciano con la realtà concreta all'interno degli intermediari finanziari. Più in generale, la banca ha un ruolo da protagonista nel sistema economico, in quanto alloca risorse (o liquidità) da situazioni di surplus economico a quelle di deficit economico. La quasi totalità delle banche occidentali (cc.dd. convenzionali), raccoglie depositi dalle famiglie e impiega tale danaro riallocandolo, sotto forma di prestito finanziario, alle imprese. Solitamente, l'intermediario bancario ottiene remunerazione dalla differenza tra i tassi attivi e i tassi passivi ${ }^{76}$.

La banca islamica, invece, pur rivestendo un ruolo fondamentale al pari di quella convenzionale, è il luogo in cui la finanza islamica trova massima espressione. È intrisa di regole e principi di diritto islamico, quale prodotto della Rivelazione coranica, risultando perciò un modello economico conforme al Verbo di Dio.

Uno dei punti di maggior discontinuità della banca islamica rispetto a quella convenzionale è dato, come già ricordato, dal divieto di ribà. Pertanto, le banche islamiche hanno un divieto espresso di remunerazione derivante dall'applicazione di un tasso d'interesse prestabilito; al contrario, è halal il rendimento di una banca quando questa prende parte concretamente a un'attività reale e se tale rendimento pecuniario è la conseguenza di una attività d'impresa. Ne consegue che la banca islamica ha un ruolo, oltre che economico, anche sociale, in quanto funge da vero e proprio gestore di fondi, mettendo al vaglio la bontà dei progetti imprenditoriali, anziché valutare la sola capacità di solvibilità del debitore come accade nel mondo bancario convenzionale. Ciò, come

risposta/chiarimento. Sul punto, cfr. F. CASTRO, Il modello islamico, cit., 2007, p. 23 s.

${ }^{76}$ R. HAMAURI, M. MAURI, Economia e finanza islamica, cit., p. 78 ss.; P. BIANCONE, La banca islamica, cit., p. 79 ss. 
sostiene autorevole dottrina77, potrebbe avere un impatto positivo sull'economia reale, evitando fenomeni speculativi presenti nel mercato bancario occidentale, anche se con una maggior esposizione della banca islamica al rischio di eventuali perdite.

Il modello di business utilizzato dalla banca islamica ha dato vita, nel corso del tempo, a numerosi costrutti contrattuali specificamente impiegati nel settore finanziario. Si tratta di una categoria, quella dei contratti finanziari, in crescente espansione e che mira alla creazione di strumenti via via più complessi dati dalla necessità prodotta dall'evoluzione delle operazioni di transazione.

Nel paragrafo che segue, verranno presi in considerazione i contratti di partecipazione, i quali si basano sul principio del Profit Loss Sharing (PLS) ${ }^{78}$.

\section{5 - I contratti di tipo partecipativo: mudàraba e mushàraka}

Al pari della disciplina della maggior parte dei contratti del mondo occidentale, le regole e i principi del diritto dei contratti islamici devono mirare alla realizzazione di interessi meritevoli di tutela ${ }^{79}$ che rispecchino, entro i vincoli sopracitati, la volontà delle parti. Tutte le tipologie contrattuali islamiche, anche i contratti di tipo partecipativo ( $v$. infra), seguono alcuni dettami che possiamo definire regole generali.

77 R. HAMAURI, M. MAURI, Economia e finanza islamica, cit., p. 79 ss.

${ }_{78} \mathrm{Si}$ tratta del sistema di condivisione dei profitti e delle perdite. Sebbene l'Islam escluda interessi nelle attività finanziarie, ciò non necessariamente significa che chi finanzia non possa guadagnare o ricavare profitti. In generale, i guadagni realizzati sono direttamente relazionati al rischio intrapreso sull'investimento (S.A. SIDDIQUI Shari'ah compliance, performance, and conversion: the case of the Dow Jones Islamic Market Index, in Chicago Journal of International Law, 2007, p. 496 ss.). Senza rischio coinvolto, il guadagno realizzato rappresenta un interesse piuttosto che un profitto. Per capire come il sistema islamico differenzi tra profitto e interesse, si deve considerare che, in un sistema capitalistico, il ritorno del capitale si concretizza nell'interesse, mentre l'imprenditore, ossia chi rischia una perdita, guadagna a profitto. Mentre l'interesse è un ritorno fisso per fornire capitale, il profitto può solo essere guadagnato dopo che viene distribuito il ritorno fisso, lavoro e capitale (nella forma di affitto, salario, etc.).

79 Cfr., sul punto, l'art. 1322, secondo comma, del Codice civile italiano, ai sensi del quale "le parti possono anche concludere contratti che non appartengono ai tipi aventi una disciplina particolare, purché siano diretti a realizzare interessi meritevoli di tutela secondo l'ordinamento giuridico". Tale norma potrebbe essere una strada per permettere una considerazione positiva della contrattualistica islamica in Italia. 
La completa informazione e la trasparenza ${ }^{80}$ sono condizioni, infatti, che le parti devono rispettare; fattori, questi, che trovano spazio con riguardo gli elementi essenziali ${ }^{81}$ del costrutto contrattuale, divenendo così un parametro per la valutazione di validità di un contratto Shari'ah compliant. Tali requisiti si estendono ben oltre il sinallagma contrattuale, governando così il procedimento sin dalle trattative ('iqad). Ne consegue l'inammissibilità di qualsivoglia ambiguità in relazione alla lingua utilizzata per redigere il contratto, all'oggetto e alla capacità dei soggetti ${ }^{82}$.

La chiarezza nella formulazione delle clausole contrattuali si riflette, divenendone un elemento essenziale, nella formazione della volontà contrattuale. Anche volendo delineare una regola generale del contratto, questo è prodotto dalla volontà delle parti, volontà, formatasi dall'incontro tra una proposta (ijab) e un'accettazione (qabul) ${ }^{83}$. Di conseguenza, la proibizione del ghàrar assume un ruolo che va al di là del mero profilo economico-finanziario della fattispecie contrattuale mirando così alla piena tutela nella formazione della volontà del proponente $\mathrm{e}$ dell'accettante $^{84}$. Da ciò deriva che il contratto deve sempre escludere elementi che possano creare uno squilibrio idoneo a produrre un'alterazione del sinallagma a favore di una delle parti. Ad esempio, la mancata conoscenza dell'oggetto, la durata del contratto, la qualità e la quantità dei beni, l'ambiguità nella terminologia, etc.

I profili accennati trovano spazio in molteplici fattispecie contrattuali, non da ultimo in ambito finanziario. Infatti, i contratti mudàraba e mushàraka vengono ampiamente utilizzati dalle banche islamiche ${ }^{85}$ per il loro carattere competitivo, al riparo da tassi di interesse e dall'incertezza vietati dal Corano ${ }^{86}$.

80 La regola della trasparenza e della completa informazione rappresentano il precipitato giuridico del divieto di ghàrar (cfr. par. 3 del presente contributo).

81 Sul punto si rimanda a T.V. RUSSO, I contratti Shari'ah compliant, cit., p. 62.

82 T.V. RUSSO, I contratti Shari'ah compliant, cit., p. $61 \mathrm{~s}$.

83 T.V. RUSSO, I contratti Shari'ah compliant, cit., p. 62.

84 T.V. RUSSO, I contratti Shari'ah compliant, cit., p. 62. È bene rimarcare che la tutela della formazione della volontà del proponente e dell'accettante è presente anche negli ordinamenti giuridici secolari. In Italia, ad esempio, il Codice civile prende in esame questo profilo, si pensi all'art. 1326.

85 Anche nel vecchio continente le fattispecie contrattuali in esame hanno fatto il loro ingresso. Ci si riferisce alla cosiddetta capitale della finanza islamica in Europa, id est Londra. Basti pensare che la Al Rayan Bank (https://www.alrayanbank.co.uk/) nel 2018 registrava un cliente su tre non musulmano contro l'uno su otto nel 2010. La borsa londinese quota circa 70 obbligazioni sukuk per un valore di oltre 700 milioni di sterline. 
Nel costrutto di credito c.d. partecipativo utilizzato dalle banche, la mushàraka87 occupa una posizione di assoluta preminenza. È dunque la forma contrattuale in cui trova accoglimento, in modo più notevole, il principio del Profit Loss Sharing88. La mushàraka è dunque una società (sarika) in cui due o più soci mettono in comune il capitale e copartecipano al business management. La banca ( $r a b b$ al-maal) è a tutti gli effetti co-proprietaria di una percentuale della compagine societaria e ha la facoltà di esercitare un potere di controllo sulle scelte di direzione del progetto imprenditoriale oggetto dell'accordo della mushàraka, oltre ad avere il diritto di intervenirvi direttamente. Sin dalle origini ${ }^{89}$, la mushàraka è stato impiegato per la condivisione del rischio in un'attività di impresa, e viene spesso accostato al Joint Venture ${ }^{90}$ del mondo contrattuale anglooccidentale.

Difatti, in tale schema contrattuale le parti partecipano allo schema di business attraverso il conferimento di capitale (beni, soldi, terreni, etc.) e condividono gli attivi e i passivi derivanti dall'esercizio dell'attività di impresa. Inoltre, i soci condividendo il proprio capitale, non sono estranei al running a business. Eventuali perdite vengono suddivise in base alla seguente regola: si tiene conto della percentuale del capitale immesso nella società e, in modo proporzionale a questo, vengono ripartite le passività.

In Irlanda, Lussemburgo e Svizzera i principi della finanza islamica sono abbracciati da tempo. L'Italia, dal canto suo, appare interessata alla finanza islamica quantomeno dal punto di vista dei possibili investimenti che soggetti appartenenti al mondo islamico potrebbero effettuare in società industriali e finanziarie del nostro Paese, nonché della raccolta di capitali che gli intermediari italiani potrebbero effettuare sia in Paesi islamici, sia dagli oltre un milione e mezzo di musulmani che oggi risiedono in Italia.

${ }^{86}$ Cfr. par. 3.

87 Il termine mushàraka (dall'arabo Sharika) implica una partnership in un'impresa o in un progetto imprenditoriale. Sul punto, cfr. P. BIANCONE, La banca islamica, cit., p. 89. Inoltre, si rimanda, per la consultazione di uno schema di mushàraka, tra le varie, a G.M. PICCINELLI, Banche islamiche, cit., p. 205 ss.

88 P. BIANCONE, La banca islamica, cit., p. 69 ss., che esamina in maniera puntuale i profili che differenziano la banca islamica dalla banca tradizionale, affermando che "il divieto di interesse ha posto alla banca islamica la questione di come rimpiazzare il meccanismo del tasso di interesse per rendere più profittevole la sua struttura operativa: il ricorso al principio della condivisione dei profitti e delle perdite (PLS) è stata la risposta a questo problema".

89 Per una sintesi storica del contratto di mushàraka si rinvia a G.M. PICCINELLI, Banche islamiche, cit., p. 120.

90 Così G.M. PICCINELLI, Banche islamiche, cit., p.133. 
Invero, come ricordato dall'AAOIFI' ${ }^{91}$, gli utili non seguono per forza il criterio imposto invece per eventuali passività. Infatti, anche secondo le best practices, è ammissibile una ripartizione degli utili che non segua necessariamente un criterio proporzionale alla percentuale del capitale conferito alla compagine economica d'impresa purché, tale profilo, sia inserito (ab initio) nel contratto, evitando così di incorrere nell'invalidità causa la proibizione di ghàrar.

Ne consegue che la percentuale di profitto non possa essere stabilita a forfait, bensì deve essere stabilita ex ante prima della conclusione del contratto. Data la diversa immissione di capitale da parte dei soci, anche la divisione percentuale delle perdite e dei profitti può essere ineguale ${ }^{92}$. Invece, ponendo l'accento sulle perdite, si è cristallizzata la regola secondo cui queste si attribuiscono obbligatoriamente in base ai conferimenti apportati nel tessuto dell'impresa.

Altro profilo che merita di essere approfondito riguarda la durata (e quindi il termine) del contratto. La dottrina prevalente ritiene che la mushàraka debba essere portata avanti sino alla piena realizzazione dell'oggetto del contratto; altri, invece, ritengono che l'accordo si possa rescindere in qualsiasi momento ovviamente previa conoscenza delle altre parti.

Questi profili estintivi del contratto creano alcuni problemi, soprattutto per quanto riguarda $\mathrm{i}$ soci che abbiano dichiarato di non apportare lavoro (sleeping partner), in quanto potrebbero non essere così incentivati a supportare al massimo la realizzazione del progetto. Come si vedrà in seguito, questo profilo è sicuramente meno marcato rispetto alla fattispecie di mudàraba.

I giuristi sono unanimemente d'accordo che il contratto in esame sia totalmente conforme alle regole shariatiche, quindi, che rientri nel novero Shari'ah Compliant.

Il mudàraba93, alla stregua della mushàraka, è una forma contrattuale partecipativa che ha un ruolo di primo piano nella compagine degli

91 Si veda AAOIFI, Accounting and Auditing Organization for Islamic Financial Institutions /in https://aaoifi.com).

92 P. BIANCONE, La banca islamica, cit., p 89 ss., il quale, riportando la dottrina prevalente, afferma che "secondo alcuni giuristi (Iman Malik, Iman Shafi e Imam Zufar), come regola generale, ogni socio dovrebbe ricevere una parte dei profitti esattamente in proporzione ai capitali da lui apportati". Tuttavia, continua dichiarando che "non è obbligatorio che l'accordo per l'attribuzione dei profitti preveda una proporzionalità al contributo di capitale".

93 Sul punto si veda P. BIANCONE, La banca islamica, cit., p. 82 ss.; T.V. RUSSO, I 
strumenti finanziari adottati dagli intermediari finanziari Shari'ah Compliant. È uno strumento di finanziamento che si sostanzia in una società in accomandita in cui il rabb al-maal (la banca) attribuisce il capitale (solitamente in mero danaro) mentre il mudarib (il cliente-imprenditore) mette a disposizione lavoro, esperienza e più in generale capacità gestionali legate all'oggetto del contratto di mudàraba.

Come avviene nella società in accomandita del diritto italiano ${ }^{94}$, l'immissione del capitale da parte del rabb al-maal non fa sorgere in capo a questo nessun diritto alla gestione dell'attività. Vi è una impermeabilità tra il concedente fondi e l'attività che viene liberamente gestita dall'imprenditore non dovendo garantire un ritorno in utili alla banca; inoltre, il mudarib, in caso di perdite, è esente da responsabilità salvo non sia provata la mancanza della diligenza media richiesta a un soggetto in quella identica situazione. In altri termini, il contratto di mudàraba è un contratto in cui una parte finanzia in capitale e l'altra gestisce il lavoro realizzando il progetto imprenditoriale accordato in fase di stipulazione contrattuale.

Per quanto riguarda il profilo attinente alla ripartizione dei profitti, questi devono essere conferiti alle parti sulla base di un sentimento di comune accordo nel momento della conclusione del contratto ${ }^{95}$, non è richiesto ai fini della validità del vincolo contrattuale che la divisione degli utili segua una logica alla pari, al contrario, è possibile che le parti abbiano cristallizzato nell'accordo percentuali diverse in apposite clausole stilate $a b$ origine $^{96}$.

Lo schema contrattuale in esame è l'espressione della massima autonomia delle parti che deve dare anima al costrutto e quindi, anche per quanto attiene il termine del contratto, le parti sono libere di agire di comune accordo ${ }^{97}$.

La critica ricorrente riguardante questa tipologia di contratto ruota attorno al problema cosiddetto di agenzia: il rabb al-maal si assume totalmente il rischio delle (eventuali) perdite, e questo ha prodotto un sentimento di profondo sospetto circa il mudarib il quale, non accollandosi il rischio di perdite, potrebbe non impiegare le necessarie energie per

contratti Shari'ah compliant, cit., p. 68 ss.

94 Disciplinata dagli artt. 2313-2322 c.c.

95 Questo profilo è emerso già nel contratto di mushàraka al fine di garantire il rispetto del principio di ghàrar (vedi supra).

96 P. BIANCONE, La banca islamica, cit., p. 85.

97 P. BIANCONE, La banca islamica, cit., p. 85. 
raggiungere la realizzazione del progetto imprenditoriale ${ }^{98}$. Infatti, la banca non si può liberare dal sopportare la perdita salvo che non riesca a provare la colpa del cliente-imprenditore.

Nonostante questo punto d'ombra, il mudàraba è anch'esso ampiamente utilizzato, e trova favore tra i giuristi islamici, i quali ne sostengono la liceità e la piena conformità shariatica. Come paradigma di legalità del contratto si tiene conto del fatto che esso era ampiamente utilizzato tanto dalla società preislamica tanto nel periodo islamico; anzi, si ritiene che l'Islam lo abbia ereditato dalle prassi commerciali in uso prima della Rivelazione ${ }^{99}$.

\section{6 - (segue) Tipologie di mudàraba}

La prassi bancario-commerciale è solita riconoscere due tipi di mudàraba, a seconda del tipo di poteri gestionali esercitati da parte del mudarib, in base ai vincoli imposti dal rabb al-maal.

In particolare, se questo ha apportato capitale in un investimento determinato, ad esempio in un'impresa alimentare, si identifica un almudarabah al muqayyadah (anche chiamato restricted mudaraba), ove il mudarib può agire in un quadro imprenditoriale/progettuale specifico. Diversamente, laddove l'accordo non preveda uno specifico progetto, quindi senza vincoli, si parla di al-mudarabah al-mutlaqah (unresticted mudaraba) ${ }^{100}$.

Anche il sinallagma contrattuale del mudaraba può essere sciolto unilateralmente, salvo l'onere di comunicazione all'altra parte: si

98 P. BIANCONE, La banca islamica, cit., p. 85.

99 G.M. PICCINELLI, Banche islamiche, cit., p. 100, il quale afferma: "Nell'Arabia del settimo secolo dopo Cristo - che già conosceva nelle sue linee essenziali il contratto di mudàraba dai tempi preislamici - l'economia era prevalentemente fondata sull'autofinanziamento e sul finanziamento diretto dal capitalista all'imprenditore. Per evitare i frequenti prestiti a tassi d'usura, v'era la pratica di stipulare contratti associativi di capitale e lavoro, detti appunto mudàraba, tra parti appartenenti normalmente al medesimo contesto tribale, i quali consentivano di mettere a profitto i capitali affidandoli ad una persona esperta e capace di portare a compimento con successo una spedizione commerciale [...] Questo sistema divenne, con la proibizione del ribà, la struttura portante del credito alle attività commerciali, e, in speciale modo, al commercio sulle lunghe distanze nel mondo islamico e ben presto, a meno di un secolo dal suo sorgere, si affacciò e si stabilì attorno all'intero bacino del Mediterraneo".

100 T.V. RUSSO, I contratti Shari'ah compliant, cit., p. 70. 
procederà alla divisione delle risorse secondo le percentuali degli utili accordate.

Dal confronto tra i contratti di mushàraka e mudàraba va rilevato come quest'ultimo rappresenti uno strumento che sbilancia l'asse dell'equilibrio a sfavore della banca, in quanto a questa vengono allocate totalmente le perdite di un'attività svolta da altri (il cliente) potendosi liberare solo nel caso in cui sia provata la negligenza dell'imprenditore. In breve, sotto il profilo della ripartizione delle perdite, i due contratti partecipativi esaminati, differiscono profondamente; il mushàraka ripartisce le perdite in modo proporzionale ai conferimenti effettuati e realizza un equilibrio tra le parti del tutto assente nel mudàraba.

Non è il solo profilo che differenzia le fattispecie: infatti, nel mushàraka, anche l'apporto di capitale e la gestione del progetto sono prerogativa di tutte le parti, le quali operano alla realizzazione del progetto; ciò non avviene minimamente nel mudàraba, ove il capitale è fornito dalla banca, la quale non può prendere parte alla gestione dell'attività di impresa che rimane quindi diritto del solo imprenditorecliente. Ne consegue che anche il diritto di proprietà sugli assets muta a seconda che si parli di mushàraka o mudàraba. Nel primo caso, tutti gli attori sono proprietari sulla base del conferimento di capitale effettuato in fase di conclusione del contratto; nel secondo caso, invece, le attività sono solo del finanziatore, quindi della banca.

\section{7 - Cenni conclusivi e prospettive future}

L'Islam, foriero di precetti etico-religiosi e normativi, è il fondamento dell'intera esistenza di circa un miliardo di persone nel mondo. La Shari'ah viene considerata, come si evince dall'analisi dei contratti partecipativi, totalmente in grado di normare non solo i rapporti di natura patrimoniale tra i fedeli musulmani, ma anche le transazioni finanziarie tra le più complesse e dalla struttura articolata. Il sistema economico islamico aspira a creare un'economia fondata sui principi e sui valori propri della Shari'ah: la ricchezza individuale diviene strumento per la realizzazione della volontà di Allah. Come nel mondo Occidentale, anche nel diritto islamico la conclusione dei contratti è finalizzata alla realizzazione degli interessi delle parti. Tuttavia, è possibile sottolineare la ratio che, in parte e da diversi fronti, è emersa in questo contributo.

La ragione economico-sociale del contratto, la causa usando classificazioni convenzionali, su cui si erge l'intero settore contrattuale islamico è il rispetto dei principi della Shari'ah, per far sì che l'operato del 
musulmano sia conforme al dettato divino cooperando alla equità sociale, allo sviluppo, alla simmetria tra le parti, limitando così la tesaurizzazione a scapito del benessere della collettività. Come si è detto, "il Corano è una guida per gli uomini sia dal punto di vista spirituale sia dal punto di vista temporale"101. Infatti, proibisce ogni genere di usura o l'applicazione di qualsiasi forma di tasso di interesse, il ribà, proibisce inoltre qualsiasi incertezza (se rilevante), qualsiasi attività di scommessa e infine vieta una serie di attività considerate appunto haram. Più in generale si potrebbe dire che il diritto islamico dei contratti e, specificamente, il diritto dei contratti Shari'ah compliant ricusi totalmente qualsiasi forma di arricchimento ingiustificato e quindi che non si regga su uno scopo haram ${ }^{102}$.

È su queste premesse che si erge un'incessante attività, da parte degli intermediari finanziari, di sperimentazione di strumenti finanziari innovativi ma in linea con i precetti shariatici. Ciò ha permesso di definire le istituzioni islamiche un sistema alternativo contrapponendolo a quello bancario convenzionale.

$\mathrm{Su}$ questa linea di senso, si pongono le forme contrattuali quali mudàraba e mushàraka, fattispecie ricostruite nei paragrafi precedenti, nelle quali l'utile ripartito tra i soci è prodotto di un contributo comune.

Nate per effettuare semplici operazioni di compravendita, queste tipologie contrattuali costituiscono la base anche per operazioni più complesse, come nel mercato assicurativo o finanziario e che ben si adattano a evoluzioni e utilizzi alternativi, o meglio, a utilizzi plasmati in base alle contingenze concrete. In breve, oltre a essere prodotti finanziari che intercettano una domanda elevata sono altresì strumenti che fungono da modello base per operazioni finanziarie più complesse.

Si evince dall'analisi condotta in questa sede sui principi e sulla teoria generale del contratto che il diritto divino islamico è capace, nonostante la sua immutabilità 103 , di stare al passo con i tempi, offrendo risposte anche in ambito contrattuale.

101 Cit. in Le Commentaire Coranique. Deuxième Partie: Le Tafsir Moderne et Contemporain, Dossier des Etudes Arabes del PISAI, n. 69, 1985, pp. 137-139.

102 Sul punto si rimanda ai paragrafi 2 e 3.

103 I diritti religiosi, tra cui si colloca anche il diritto islamico, sono immutabili. Con questa espressione si intende definire una tendenziale rigidità dovuta alla cornice in cui il diritto religioso si snoda e si sviluppa. La dottrina sul punto è unanime nel ritenere che la portata dell'immutabilità del diritto religioso non sia di fatto assoluta bensì relativa. La Rivelazione è immutabile per definizione ma le interpretazioni cambiano al fine di dare delle risposte che siano tendenzialmente in linea con il periodo storico. Pertanto, i principi e valori contenuti nei testi sacri sono immutabili nell'essenza ma mutabili 
È indubbio che la Rivelazione non abbia previsto forme contrattuali applicabili al campo finanziario e bancario ma è stata comunque in grado di offrire risposte ai propri adepti in un settore apparentemente così lontano dalla religione, i.e. il mondo finanziario. Sono stati presi in considerazione, in questo contributo, tra le varie forme esistenti, i contratti partecipativi che sono sicuramente tra i modelli più utilizzati nella prassi e che godono di un totale e indiscusso consenso da parte delle scuole giuridiche islamiche. Volendo evidenziare il profilo che funge da minimo comun denominatore delle due fattispecie è possibile affermare quindi che tanto il contratto di mushàraka quanto il contratto di mudàraba sono conformi alla Shari'ah; in più sono un possibile esempio di come la legge religiosa sia in grado di offrire soluzioni adeguate in ogni settore della vita dei fedeli, non da ultimo il settore dell'economia, confermando così il carattere di completezza che connota il diritto islamico.

In ultima battuta, rivolgendo lo sguardo verso l'ordinamento italiano è possibile affermare che questo non abbia, a oggi, conosciuto alcuna esperienza in ambito finanziario islamico e di conseguenza neanche con i contratti islamici di tipo partecipativo qui trattati.

In altre parole, le realtà finanziarie e bancarie italiane non hanno concretizzato, se non incidentalmente ${ }^{104}$, proposte sistematiche e unitarie volte a dischiudere le molteplici possibilità derivanti dall'introduzione della finanza islamica nell'ordinamento bancario e, più in generale, finanziario italiano. Opportunità che si muovono su diversi piani che, in questo contributo, hanno fatto da sfondo, ossia la possibilità di un'integrazione (anche) finanziaria dei fedeli musulmani e la possibilità per l'Italia stessa di attirare investimenti da Paesi come quelli del Golfo che attualmente possiedono ingenti patrimoni che potrebbero rappresentare un vero e proprio detonatore per la ripresa dell'economia italiana.

Potrebbe apparire utile guardare il fenomeno allargando la lente prospettica: analizzare in modo puntuale gli ordinamenti stranieri in cui la finanza islamica è riuscita ad attecchire, utilizzando questi come veri e propri legal case. Sicuramente un posto di primo piano è ricoperto del

nell'applicazione. Per un'analisi dettagliata sulle caratteristiche dei diritti religiosi si richiama S. FERRARI, Lo spirito dei diritti religiosi. Ebraismo, cristianesimo e islam a confronto, il Mulino, Bologna, 2002, p. 22 ss.

${ }^{104} \mathrm{Ci}$ si riferisce alla proposta di legge titolata "Disposizioni concernenti il trattamento fiscale delle operazioni di finanza islamica". Sul punto si rimanda al documento consultabile al seguente sito: http://documenti.camera.it/leg18/pdl/pdf/leg.18.pdl.camera.201.18PDL000 2750.pdf. 
Regno Unito che ha esperienze in materia di finanza islamica sia sotto forma di islamic windows sia sotto forma di (total) islamic banking105. L'ordinamento inglese ha già affrontato i vari punti di criticità tra sistema islamico e sistema giuridico interno, superando, di fatto, gli apparenti nodi gordiani imperanti nell'annosa questione dell'affermarsi della finanza islamica. Sarebbe opportuno, quindi, nell'ottica di chi scrive, richiamare quella parte della dottrina ${ }^{106}$ che sostiene che, al netto dell'esperienza inglese, la policy britannica potrebbe essere la strada percorribile anche per l'Italia, permettendo così l'ingresso degli strumenti Shari'ah compliant, tra cui i contratti di mushàraka e mudàraba. Infatti, il sistema giuridico e fiscale del Regno Unito ha usato come bussola orientativa due elementi. Il primo legato al fatto che l'esecutività legale di un contratto non deve dipendere dalla compatibilità con la Shari'ah; il secondo aspetto, invece, è legato alla libertà riconosciuta ai consociati di organizzare la propria sfera giuridica purché non in contrasto con i principi dell'ordinamento statale. Fra le varie vie percorribili, quest'ultimo assunto richiama la lettera dell'art. 1322 del Codice civile italiano rubricato 'autonomia contrattuale', il quale potrebbe rappresentare un grimaldello per l'introduzione dei contratti Shari'ah compliant in Italia unitamente a un intervento ad hoc del Legislatore volto a rimuovere gli ostacoli di natura fiscale.

Uno studio comparato quindi si rivela lo strumento più idoneo per prospettare una strada per affermare le potenzialità inespresse della finanza islamica in contesto italiano.

105 Per un'analisi puntuale sulle banche islamiche britanniche si rimanda a $\mathbf{P}$. BIANCONE, La banca islamica, cit., p. 25 ss. Inoltre, si richiama anche l'esperienza della Germania che è sicuramente più recente e che presenta anch'essa possibili spunti d'indagine. Per ulteriori approfondimenti si rinvia a P. BIANCONE, La banca islamica, cit., p. 36 ss.

${ }^{106}$ F. MIGLIETTA, P.P. RAMPINO, Gli strumenti di finanza islamica, cit., p. 68 ss. 